\title{
Architecture of the Organic.Edunet Web Portal
}

\author{
Nikos Manouselis ${ }^{1}$, Kostas Kastrantas ${ }^{1}$, Salvador Sanchez-Alonso ${ }^{2}$, Jesús Cáceres ${ }^{2}$, \\ Hannes Ebner ${ }^{3}$, Matthias Palmer ${ }^{3}$, Ambjorn Naeve $^{3}$ \\ ${ }^{1}$ Greek Research \& Technology Network (GRNET S.A.), Greece \\ nikosm@ieee.org; kkastrad@grnet.gr \\ ${ }^{2}$ University of Alcalá, Spain \\ \{salvador.sanchez, jesus.caceres\}@uah.es \\ ${ }^{3}$ Royal Institute of Technology (KTH), Stockholm, Sweden \\ $\{$ hebner, matthias, amb\}@csc.kth.se
}

\begin{abstract}
The use of Semantic Web technologies in educational Web portals has been reported to facilitate users' search, access, and retrieval of learning resources. To achieve this, a number of different architectural components and services need to be harmonically combined and implemented. This paper presents how this issue is dealt with in the context of large-scale case study. More specifically, it describes the architecture behind the Organic.Edunet Web portal that aims to provide access to a federation of learning repositories with learning resources on agricultural topics. The various components of the architecture are presented and the supporting technologies are explained. In addition, the paper focuses on how Semantic Web technologies are being adopted, specialized, and put in practice in order to facilitate ontology-facilitated sharing and reusing of learning resources.
\end{abstract}

Keywords: learning object, Web portal, Semantic Web, organic farming

\section{Introduction}

Following their introduction and commercial growth after 2000, Web portals have lately attracted increased research interest that focuses on a variety of aspects such as their business models, interface design, technical development, or their quality (Mahadevan, 2000; Tatnall, 2005a; Moraga et al., 2006; Tatnall, 2007). The term Web portal has been initially used to refer to well-known internet search and navigation sites that provided a starting point for web visitors to explore and access information on the World Wide Web (Warner, 1999; Winkler, 2001). A Web portal can be generally viewed as a single, distilled view of information from various sources that integrates information, content, and other software services or applications (Averweg, 2007). Therefore, today Web portals can be simply defined as gateways to information and services from multiple sources, and their continuous development has been highlighted by relevant publications (Tatnall, 2005b).

A type of Web portals with particular interest are educational ones (Conceicao et al., 2003; Boff et al., 2006). Educational Web portals generally serve as gateways to information and services of some learning or teaching relevance and may cover a variety of types. They range from institutional Web portals that provide access to course listings and institutional information (such as Ethridge et al, 2000), to community portls that serve the needs of particular communities of learning and practice (such as DeSanctis et al., 2001; Luke et al., 2004). One category of educational portals that have recently received considerable interest (Neven \& Duval, 2002; Richards et al., 2002; Hatala et al., 2004) is that of Web portals that provide access to some organized collection of learning resources. These portals usually facilitate users' access to the content in one or more learning repositories - that is, to database systems that facilitate the storage, location and retrieval of learning resources (Holden, 2003). Popular examples include both independent learning resources' portals such as MERLOT (http://www.merlot.org) and Teachers' Domain (http://www.teachersdomain.org/), as well as portals that list or aggregate learning resources from various other sources (e.g. other portals or repositories) such as OERCommons (http://www.oercommons.org). 
Richards et al. (2002) stress that Web portals with learning resources may offer a wide variety of services based on what they seek to give to the user community behind them, although the more common are those aimed at facilitating users' search, access, and retrieval of the resources. For this purpose, they include services that will facilitate these processes, utilizing different types of user-related information (such as personal preferences) or resource-related information (such as the learning resource characteristics). One of the most recent trends in portal development is the use of Semantic Web technologies (Maedche et al., 2001). Semantic Web is an evolving extension of the World Wide Web (WWW) in which web content can be expressed not only in natural language, but also in a format that can be read and processed by software systems, thus permitting them to find, share and integrate information more easily (Berners-Lee, 1998). Numerous applications and case studies of Semantic Web technologies (e.g. ontologies for annotating information and expressing its semantics in a machineprocessable manner) have been reported during the past few years. For instance, the World Wide Web Consortium (W3C) reports on several systems that have been put in production in existing organizations, as well as a number of commercial products (http://esw.w3.org/topic/CommercialProducts). Yet, the Semantic Web technologies have not so far reached the wide public. Some of the experts in the field claim that the reason is that large-scale applications, serving the needs of large user communities, have not been delivered yet (Shadbolt et al., 2006). To further illustrate their potential (and especially for Web portals), there is a need for implementing state-of-the-art Semantic Web technologies in largescale applications. In the context of educational Web portals, this involves the semantic annotation of big collections of learning resources and their access and use from existing communities of users.

This paper aims to contribute to this development by presenting such a large-scale implementation effort. More specifically, it discusses how semantic annotation and Semantic Web technologies are being adopted, specialized, and put in practice in order to set up a technical infrastructure that will facilitate sharing and reusing of learning resources for an educational Web portal. The case study is the Organic.Edunet Web portal, a portal that serves the needs of learning and teaching communities of the agricultural sector, by facilitating their access to a network (also called a federation) of learning repositories with learning resources on Organic Agriculture (OA) and Agroecology (AE) topics.

The paper is structured as it follows. First, a short review of the way Semantic Web approaches are being implemented in similar applications is carried out. A description of the Organic.Edunet initiative is given, and the rationale for developing the Organic.Edunet Web portal is outlined. The main part of the paper focuses on the description of the technical architecture of the Web portal, and on the way Semantic Web technologies are implemented in it. A discussion of perceived benefits and potential challenges is later carried out, to finally provide the main conclusions of this work.

\section{Background}

\subsection{Educational Semantic Web}

From its initial conception around 1989 (Berners-Lee, 1998), the WWW (or simply, the Web) has been designed as an information space, with the goal that it should be useful not only for human-to-human communication, but also for machines that would be able to mediate and help. As Berners-Lee reports, one of the major obstacles to this has been the fact that most information on the Web is designed for human consumption, and even if it was derived from a very well specified database, the structure of the data is not evident to an automated software system browsing the Web. On the contrary, in his vision of the Semantic Web, data 
recovery for a particular context of use should be a routine, automated process. This is the reason why the empowering role of Semantic Web technologies has already been acknowledged in various contexts, such as education and training (Aroyo \& Dimitrova, 2006). In this new paradigm, data would be specifically oriented to machine consumption by means of formal descriptions based on the existence and wide availability of ontologies, knowledge models of a given domain. Ontologies, which can be defined as collections of concepts representing domain-specific entities, the relationships between those concepts, and the range of admissible values for each concept (Daraselia et al., 2004) are in fact the key element of the Semantic Web. Ontologies serve as knowledge models for each particular domain of science, thus allowing to unambiguously represent, refer, and describe entities in that domain, and serving as the basis for interoperability and common understanding under formal and strict semantics.

Since the Web is becoming a popular educational medium at schools, universities and professional training institutions, a prominent new stream of research on the Educational Semantic Web has been established. Research studies already report semantic-based annotation and sharing of learning resources. For example, Forte et al. (1999) report on the principles underlying the semantic and pedagogic interoperability mechanisms built in the European Knowledge Pool System, a distributed repository of learning objects developed by the European research project ARIADNE (http://www.ariadne-eu.org).

In addition, Soto et al. (2007) designed an ontology schema capable to bring more flexibility to the description of the entities stored in semantic learning object repositories and, at the same time, to facilitate automated functions and task delegation to agents. Furthermore, Sicilia et al. (2005) describe the design of a learning object repository approach to what they called "semantic lifecycle" and illustrate thus through the concrete architecture of a semantic learning object repository prototype.

Moreover, semantic web applications are becoming more and more usual in education \& training contexts. Sancho et al. (2005) for instance, applied these technologies to e-learning personalization by combining the information provided by ontologies, and the user profile, to create personalized units of learning. Santos et al. (2006) described an approach to promote interoperability among heterogeneous agents that are part of an educational portal. Their main contribution was to provide a means for social agents to communicate with agents outside its original scope through the use of semantic web technologies. Other implementations of Semantic Web technologies in educational portals also exist in the literature (Woukeu et al., 2003; Tane et al., 2004; Moreale \& Vargas-Vera, 2004; Verdejo et al., 2004; Kotzinos et al., 2005).

To further illustrate the potential of the Educational Semantic Web, there is a need for implementing state-of-the-art Semantic Web technologies in large-scale applications that involve the semantic annotation of big collections of learning resources and their access and use from existing communities of users.

\subsection{Organic.Edunet}

To further promote the familiarization of consumers with the benefits of $\mathrm{OA}$ and $\mathrm{AE}$ - for their own health as well as for the benefits of the environment - the most dynamic consumer groups have to be properly educated. Young people at all stages of formal education have to be carefully approached through relevant educational programs in the curricula of all kinds of educational institutions, from elementary schools to relevant university departments. But apart from raising the awareness and education level of consumers, agricultural professionals must also be properly educated. By "agricultural professionals" we refer to the different types of future agricultural experts (e.g. natural production experts, veterinary experts, agricultural economists, extension officers, etc.), who study in agricultural universities around Europe, 
and who should be provided with a wide range of information related to OA and $\mathrm{AE}$ theories, methods, practices, and economic/environmental impacts.

Both groups (pupils and young agricultural students) constitute user groups of high importance. Children constitute tomorrow's consumers, and they have to be properly approached and educated so that their nutritional, as well as their ecological and environmental awareness are developed. Students of agricultural universities constitute tomorrow's agricultural professionals. They are expected to guide farmers through the adoption of $\mathrm{OA}$ and $\mathrm{AE}$ principles, or to serve themselves as the next generation of farmers/producers. Therefore, these two user groups have to be carefully approached through publicly available, quality, and multilingual educational content.

In this direction, the Organic.Edunet initiative (http://www.organic-edunet.eu), a European project that is funded by the $e$ Contentplus Programme and involves 15 partners from 10 countries, aims to facilitate access, usage and exploitation of digital educational content related to $\mathrm{OA}$ and AE. Organic.Edunet will deploy a multilingual online federation of learning repositories, populated with quality content from various content producers. In addition, it will deploy a multilingual online environment (the Organic.Edunet Web portal) that will facilitate end-users' search, retrieval, access and use of the content in the learning repositories. In this way, digital content resources that can be used to educate European youth about the benefits of $\mathrm{OA}$ and $\mathrm{AE}$, will become easily accessible, usable and exploitable.

To achieve its aims, Organic.Edunet adopts state-of-art technologies that have been developed and tested in several research initiatives, but have yet to be proven in a real-life context. A characteristic example involves the implementation of Semantic Web technologies that have been previously developed in the context of the "LUISA: Learning Content Management System Using Innovative Semantic Web Services Architecture" EU project (http://www.luisa-project.eu/). The main characteristics of the LUISA architecture are its service-orientation and the built-in capabilities for semantic querying. For this purpose, semantic Web Services are involved, reusing the EU framework WSMO (http://www.wsmo.org/) for the brokering of multiple repositories. WSMO (which stands for Web Service Modeling Ontology) provides ontological specifications for the core elements of Semantic Web services. Taking the Web Service Modeling Framework (WSMF) as reference, WSMO defines four different elements for describing semantic Web Services: ontologies that provide the terminology used by other elements, goals that define the problems that should be solved by Web Services, Web Services descriptions that define various aspects of a Web Service, and finally mediators which bypass interpretability problems. In the following section, we describe the overall architecture of the Organic.Edunet infrastructure, and how technologies such as the ones adopted from LUISA are engaged.

\section{TO PUT ABOUT HERE Figure 1}

\section{Organic.Edunet Infrastructure}

\subsection{Overall architecture}

The overall architecture of Organic.Edunet is illustrated in Figure 1. The main elements of this architecture are the following:

- Learning Repository Management Module: includes the suite of tools that the Organic.Edunet content providers will use to create a digital collection of learning resources, to describe resources with appropriate metadata, and to publish resources in 
their own learning repository. Overall, six learning repositories are expected to be set up by the Organic.Edunet content providers (namely the Bio@gro, ENOAT, ECOLOGICA/COMPASS, Intute, School, and Public Resources ones).

- Learning Resource Exchange Module: concerns the connection of the Organic.Edunet federation with other federations of learning repositories, using open standards and specifications for the exchange of search queries and the harvesting of metadata. Organic.Edunet is expected to be connected with two external federations; the Learning Resource Exchange (LRE) of the European Schoolnet (http://lre.eun.org) and the ARIADNE Foundation (http://www.ariadne-eu.org/).

- Semantic Services Module: it is the core of the Semantic Web technologies' application in the architecture, and supports the semantically-enabled services that the Organic.Edunet Web portal will offer, by reasoning upon a number of integrated ontologies.

- Web Portal Module: refers to the end-user visible parts of the whole infrastructure, allowing users (including school teachers and pupils, university teachers and students, researchers etc.) to search, locate, retrieve and access learning resources on $\mathrm{OA}$ and $\mathrm{AE}$ throughout the whole Organic.Edunet federation.

Each module is further detailed in the paragraphs that follow.

\section{TO PUT ABOUT HERE Figure 2}

\subsection{Learning Repository Management Module}

This module deals with the way content producers organize, annotate and publish learning resources and metadata in an Organic.Edunet repository. As illustrated in Figure 2, each of the Organic.Edunet content providers is expected to collect and annotate its learning resources, according to a multilingual application profile of the IEEE Learning Object Metadata (LOM) standard (LTSC, 2002). Two existing software tools are being adapted and integrated for this purpose:

- A configurable metadata editor built upon the code-library SHAME (available as Open Source at http://shame.sourceforge.net). With this code-library application programmers can develop flexible and easily extensible annotation tools for Semantic Web-based metadata. SHAME implements the Annotation Profile Model (Palmér et al., 2007a; Palmér et al., 2007b). This model is a configuration mechanism for annotation of metadata and leaves the question of metadata standard compliance up to a metadata expert and not to the application developer.

- The electronic portfolio system Confolio (http://www.confolio.org) that allows the flexible management of folder-based repository interfaces.

The content providers will use the integration of the SHAME editor and the Confolio tool in order to upload (if desired) their resources and the associated metadata into a learning repository that is called SCAM (Standardized Contextualized Access to Metadata), an Open Source Semantic Web repository solution for learning resources (Palmér et al., 2004). Figure 3 presents how a SCAM repository is accessed, using a combination of technologies.

\section{TO PUT ABOUT HERE Figure 3}


The repository backend is resource-oriented and will store its metadata according to a Resource Description Framework (RDF, www.w3.org/RDF/) representation of the Organic.Edunet IEEE LOM application profile. The repository provides a range of connection interfaces, allowing the most appropriate to be chosen for each situation. An interface which exposes the repository closest to the internal representation is the REST (Representational State Transfer), a resource-based software architecture building fully on top of well established standards such as the HTTP protocol (Fielding, 2004). This makes it very easy to build interactive web applications on top of this interface.

The Confolio repository front-end builds on top of the REST-based web services exposed by the repository and an AJAX (Asynchronous JavaScript and XML) toolkit, which enables cross-browser compatibility and operating system independent application. The basic operations of Confolio can be separated in two groups: administrative (e.g. creation of new portfolios of learning resources) and end-user (e.g. creation of folders and description of resources using the SHAME metadata editor).

Using the Learning Repository Management Module, Organic.Edunet content providers may collect resources, annotate them using metadata conforming to the developed application profile, reviewing and approving resources, and then releasing resources for publication. Then, the metadata of the resources stored in a particular Organic.Edunet repository are (a) made available for harvesting from the Semantic Services Module and (b) made available for harvesting and/or search federation to external federations.

\subsection{Learning Resources Exchange Module}

The Learning Resources Exchange Module allows for the communication of the Organic.Edunet repositories with external federations. Organic.Edunet will aim at the connection with the LRE and ARIADNE federations by adopting two widely used protocols and specifications:

- For communicating with ARIADNE: the Open Access Initiative Protocol for Metadata Harvesting (OAI-PHM, http://www.openarchives.org/OAI/ openarchivesprotocol.html) for making metadata available for harvesting from the ARIADNE services.

- For communicating with LRE: the Simple Query Interface (SQI, http://www.prolearnproject.org/lori) for serving/exchanging queries with the LRE services.

Metadata is transformed from its RDF representation into an XML representation, in order to be available for the external federations. Additional possibilities also exist for further interconnecting the Organic.Edunet repositories, due to their SCAM basis, e.g. the SPARQL Protocol and RDF Query Language - a W3C standardized query language and protocol for accessing RDF data (http://www.w3.org/TR/rdf-sparql-query/).

\section{TO PUT ABOUT HERE Figure 4}

\subsection{Semantic Services Module}

The Semantic Services Module is the core engine behind the Organic.Edunet Web portal that allows offering users with semantic search capabilities. To support this, it is based on a semantic representation of the learning resources' metadata, as well as a number of ontologies that are engaged during search queries to provide reasoning capabilities. More specifically, metadata is transformed into an ontological representation inside a sub-module called LOMR. The LOMR (standing for Learning Object Metadata Repository) is not itself a metadata repository but rather a framework which provides Web Service interfaces to any given, "real" 
learning object repository. LOMR instances allow developers to select the best repository implementation for a given application need, enabling specialized components, such as custom query resolvers and result composers, to benefit from the availability of different, heterogeneous LOMR instances. LOMR main features include the storage of learning object metadata in semantic format, the provision of a service-oriented interface and the import of metadata in non-semantic formats, among others.

In addition, LOMR offers Semantic Web services to the Web Portal Module, following WSMO. It uses the Web Services Modeling Language (WSML, http://www.wsmo.org/wsml/) in order to provide formal syntax and semantics for WSMO, since it is richer in reasoning capabilities than the OWL Web ontology language (http://www.w3.org/TR/owl-features/) recommended by the $\mathrm{W} 3 \mathrm{C}$. Interoperability can be easily achieved through translating WSML to OWL through open source tools that are publicly available, such as the Web Service Modeling Toolkit (WSMT, http://wsmt.sourceforge.net) and WSMO Studio (WSMO4J).

As a starting point, three ontologies are expected to be used by the Semantic Services Module. The first ontology will represent the domain area (OA and $\mathrm{AE})$. It will serve all subject classification purposes, as well as allow for reasoning related to the semantics of the $\mathrm{OA}$ and $\mathrm{AE}$ concepts themselves. For example, searching for resources that have been classified using some concepts or terms related to the ones that the user has initially indicated. The popular AGROVOC (http://www.fao.org/aims/ag_intro.htm) ontology of FAO will be used as a basis. The second ontology will be a geographical one. It will help reasoning related to the geographical origin and/or coverage of resources and their associated languages. For instance, it may allow users from a particular geographical region to search for resources in languages that have been indicated as related to the particular region, even if this has not been indicated in the initial search query. The third ontology will be representing IEEE LOM. It is expected to allow reasoning related to semantics of the LOM structure itself, such as searching for information in other elements than the ones that a user has initially indicated.

In LOMR, metadata will be harvested from the individual Organic.Edunet repositories using an appropriate harvesting mechanism. As Figure 3 shows, the RDF representations stored in the SCAM repositories will be converted to the WSML representation that LOMR requires. Once all the metadata information is stored in the LOMR repository in the formal, ontologybased format, LOMR will be able to expose various functionalities through semantic Web Services (described according to WSMO), allowing a wide variety of interactions with the Organic.Edunet Web portal.

\section{TO PUT ABOUT HERE Figure 5}

\subsection{Web Portal Module}

The final module of the Organic.Edunet architecture is the Web Portal one. It actually comprises the online environment that will interact with the various user roles (school teachers \& pupils, university teachers and students). For this purpose, it entails a role-filtering mechanism that will allow each user category to be presented with a user interface tailored to its specific needs. Apart from allowing users to semantically search and retrieve learning resources using the Semantic Services Module, the Web Portal Module will also provide the users with the option of evaluating/rating learning resources. Multi-dimensional numerical evaluations will be stored in appropriately defined evaluative metadata (Vuorikari et al., 2008). Then, they will give input to a collaborative filtering mechanism that will recommend 
users to look at resources that other users with similar preferences liked in the past (Manouselis \& Costopoulou, 2007).

\section{TO PUT ABOUT HERE Figure 6}

\section{Discussion}

\subsection{Benefits}

As it has been described in the presentation of the Organic.Edunet portal architecture, there is a number of benefits expected from the adoption of Semantic Web technologies. The following paragraphs will go into more detail on these benefits.

The use of ontologies for the classification of learning resources will allow the refinement and expansion of queries. Users currently have to rely on keyword-based searches: for instance, a teacher looking for learning resources on the advantages of the use of organic fertilizers might try something like "advantages organic fertilizers" in Google (or in any other keyword-based search engine). A search on these keywords would return results containing either the terms "organic" or "fertilizer" or "advantage" or a combination of them, but many of them would be seen as non appropriate for most users. An example on the kind of (inadequate) resources that might be retrieved with this method -traditional keyword-based searches- would be the following:

- Commercial information on products by companies selling organic fertilizers.

- Resources on the advantages of non-organic fertilizers (matching the three keywords used for the search).

- Resources criticizing the use of organic fertilizers and discouraging users on its application due to their low efficiency and high prices.

- Resources on the elaboration of organic yoghurt (as they would match to at least one of the keywords provided).

Contrary to these examples, the use of ontologies for the description of materials would force the search engine to stick to strict-matching criteria to unambiguous definitions. It would also allow to search only educational-oriented materials explicitly annotated with the predicate "explains something" and related to organic fertilizers. This would allow users to find just those learning resources explaining the advantages of the use of organic fertilizers and not criticizing them, and would allow users avoid suggestions for learning resources on other topics.

In addition, the use of ontologies will further enhance the search and browsing services offered to the users. More specifically, users will be able to browse through learning resources by selecting concepts of the ontologies used, together with an expression of their relationships. In a learning objects portal on organic farming, these technologies would help to easily access similar materials to a given one, as the relationships in the ontology would provide the ability to navigate from one instance to another. An example would be a search on learning objects about organic pest control, which would return e.g. a case study on the use of several types of insecticide-fungicide dust for use on fruit trees. Thus, portal users could navigate from the relationship from this object to fruit trees, and find e.g. learning objects on the commercialization of organic apples, or even lectures on organic fungicides applicable only to specific geographical regions.

Learning resources in current public repositories often have a high variability in their characterizations: from anything in digital format to well-defined educational oriented 
learning materials including metadata conformant to the IEEE LOM standard (McGreal, 2004). The description of all the knowledge about the domain of learning objects in the form of an ontology, and the use of this ontology as the basis for a learning object portal on organic farming, would provide the portal with the flexibility necessary to seamlessly accommodate different conceptualizations. It would also provide the ability to interact with external systems, even if each of these systems have a different understanding of what a learning object is, how their metadata should look like, etc. This model would eventually provide the users with a number of different functionalities, adapted to each particular concept of learning object, and not necessarily restricted by only one of these conceptualizations, applying technologies already in practice (Soto et al., 2007).

\subsection{Challenges}

Apart from the benefits, a number of challenges have also to be dealt with during the implementation of the Organic.Edunet architecture:

- The process of selecting, developing, and specifying the ontologies to be used (especially as far as domain-dependent ones such as the OA \& AE ontology are concerned) is demanding and time-consuming, and needs the help of a number of experts from different disciplines. To make all the experts reach agreements is not always straight-forward (Sánchez-Alonso et al., 2008.).

- The process of engineering a new ontology often implies checking the new knowledge against the commonsense knowledge and general terms in an upper ontology. This process, which has to be carefully carried out, can be summarized in four iterative phases as described by Sánchez-Alonso \& García (2006): (1) find one or several terms that subsume the category under consideration, (2) check if the mapping is consistent with the rest of the subsumers inside the upper ontology, (3) provide appropriate predicates to characterize the new category, and (4) edit it in an ontology editor to come up with the final formal version.

- Even though semantic web technologies are attractive and promise many benefits, they are not, unfortunately, ready for production use yet. Ontology-management systems hardly support the large ontologies needed for most production environments, and thus should be preferably used for research and experimentation purposes. A good example of the lack of maturity of these technologies are the APIs for ontology persistence in Java available today: Jena, Sesame and Protégé's persistence APIs find many difficulties in managing medium to large sized ontologies.

- Although the Organic.Edunet portal is based on a distributed architecture, the semantic module calls for a centralized repository that harvests the data from all repositories in the federation. Even though the harvesting tests carried out so far have shown good results, scalability, size and performance issues might arise as the project progresses and have an impact in the development.

\section{Conclusions}

To further illustrate the potential of Semantic Web technologies in Web portal applications, experiences from large-scale implementations are required. Especially in the case of Web portals that provide access to learning resources, the implementation of Web portals with services that are based on Semantic Web technologies that will be tested semantically annotating large collections of learning resources, and by being accessed and used from communities of users with numerous members. In this direction, this paper presented a largescale implementation effort that engages Semantic Web in order to set up a technical infrastructure that will facilitate sharing and reusing of learning resources for the agricultural 
domain. The next steps of this work concern reporting the results from the actual implementation, deployment, and initial testing of the technologies that are integrated in the Organic.Edunet Web portal.

\section{Acknowledgment}

The work presented in this paper has been funded with support by the European Commission, and more specifically the project No ECP-2006-EDU-410012 "Organic.Edunet: A Multilingual Federation of Learning Repositories with Quality Content for the Awareness and Education of European Youth about Organic Agriculture and Agroecology" of the eContentplus Programme.

\section{References}

Aroyo, L., Denaux, R., Dimitrova, V., \& Pye, M. (2006). Interactive Ontology-Based User Knowledge Acquisition: A Case Study. Lecture notes in computer science, 4011, 560.

Averweg U. (2007), Portal Technologies and Executive Information Systems Implementation, in A. Tatnall (Ed.), The Encyclopedia of Portal Technology and Applications, Hershey, PA: Idea Group Publishing.

Berners-Lee, T. (1998). Semantic Web Road Map. W3C Design Issues. URL http://www. w3. org/DesignIssues/Semantic. html, Oct.

Boff E., Rizzon Santos E., Vicari R.M., "Social Agents to Improve Collaboration on an Educational Portal," Sixth IEEE International Conference on Advanced Learning Technologies (ICALT'06), IEEE Computer Press, 896-900, 2006.

Conceicao S., Sherry L., Gibson D., Amenta-Shin, G.,'Managing Digital Resources for an Urban Education Portal". in Proc. of World Conference on Educational Multimedia, Hypermedia and Telecommunications (ED-MEDIA 2003), Honolulu, Hawaii, June 23-28, 2003.

Daraselia, N., Yuryev, A., Egorov, S., Novichkova, S., Nikitin, A., \& Mazo, I. (2004). Extracting human protein interactions from MEDLINE using a full-sentence parser, 20, 604-611. Oxford Univ Press.

DeSanctis G., Wright M., Jiang L., "Building A Global Learning Community", Communications of the ACM, 44 (12), 80 - 82, December 2001.

EC (2004), European Action Plan for Organic Food and Farming, COM 415, Brussels 10 June 2004.

Ethridge R.R., Hadden C.M., Smith M.P., "Building a Personalized Education Portal: Get a Behind-the-Scenes Look at LSU's Award-Winning System", Educause Quarterly, 23(3), 12-19, 2000.

Fielding, R. T. (2000). Chapter 5: Representational State Transfer (REST), Architectural Styles and the Design of Network-based Software Architectures, Dissertation.

Forte, E., Haenni, F., Warkentyne, K., Duval, E., Cardinaels, K., Vervaet, E., et al. (1999). Semantic and pedagogic interoperability mechanisms in the ARIADNE educational repository. SIGMOD Rec., 28(1), 20-25.

Hatala M., Richards G., Eap T., Willms J., "The interoperability of learning object repositories and services: standards, implementations and lessons learned", in Proc. of the 13th International World Wide Web Conference, New York, NY, USA, 19-27, 2004.

Holden, C. (2003). From Local Challenges to a Global Community: Learning Repositories and the Global Learning Repositories Summit. Version 1.0, Academic ADL Co-Lab, November 11.

Kotzinos D., Pediaditaki S., Apostolidis A., Athanasis N., Christophides V., "Online curriculum on the semantic Web: the CSD-UoC portal for peer-to-peer e-learning", in 
Proc. of the 14th International Conference on World Wide Web, Chiba, Japan, 307 $314,2005$.

LTSC (2002). IEEE Standard for Learning Object Metadata, 1484.12.1-2002. IEEE Learning Technology Standards Committee, 2002.

Luke R., Clement A., Terada R., Bortolussi D., Booth C., Brooks D., Christ D., "The promise and perils of a participatory approach to developing an open source community learning network", in Proc. of the 8th Conference on Participatory Design on "Artful integration: interweaving media, materials and practices", 11 - 19, Toronto, Ontario, Canada, 2004.

Maedche, A., Staab, S., Stojanovic, N., Studer, R., \& Sure, Y. (2001). SEAL - A framework for developing SEmantic Web PortALs. Lecture Notes in Computer Science, 2097(122), 46.

Mahadevan B., "Business Models for Internet based E-Commerce: An Anatomy", California Management Review, 42(4), 55-69, 2000.

Manouselis N., Costopoulou C. (2007). Experimental Analysis of Design Choices in MultiAttribute Utility Collaborative Filtering, International Journal of Pattern Recognition and Artificial Intelligence (IJPRAI), Special Issue on Personalization Techniques for Recommender Systems and Intelligent User Interfaces, 21(2), 311-331.

McGreal, R. (2004). Learning Objects: A Practical Definition. International Journal of Instructional Technology and Distance Learning, 1(9), 21-32.

Moraga A., Calero C., Piattini M., "Comparing different quality models for portals", Online Information Review, 30 (5), 555-568, 2006.

Moreale E., Vargas-Vera M., "Semantic Services in e-Learning: an Argumentation Case Study", Educational Technology \& Society, 7 (4), 112-128, 2004.

Neven F., Duval E., "Reusable learning objects: a survey of LOM-based repositories", in Proc. of the 10th ACM International Conference on Multimedia, Juan-les-Pins, France, $291-294,2002$.

Palmér M, Enoksson F, Nilsson M, Naeve A (2007). Annotation Profiles: Configuring forms to edit RDF. In: Proceedings of the Dublin Core Metadata Conference. DCMI Conference Papers, United States.

Palmér, M., Enoksson, F., Naeve, A (2007). LUISA deliverable 3.2: Annotation Profile Specification,.

Palmér, M., Naeve, A., Paulsson, F., (2004), The SCAM-framework - helping applications to store and access metadata on the semantic web, Proceedings of the First European Semantic Web Symposium (ESWS 2004), Heraklion, Greece, May, 2004, Springer, ISBN 3-540-21999-4.

Richards, G., McGreal, R., Hatala, M., \& Friesen, N. (2002). The Evolution of Learning Object Repository Technologies: Portals for On-line Objects for Learning. Journal of Distance Education, 17(3), 67-79.

Sánchez-Alonso S., Cáceres J., Holm A.S., Lieblein G., Breland T.A., Mills R.A., Manouselis N., "Engineering an ontology on organic agriculture and agroecology: the case of the Organic.Edunet project", in Proc. of the World Conference on Agricultural Information and IT (IAALD AFITA WCCA 2008), Tokyo, Japan, 24 - 27 August, 2008.

Sánchez-Alonso, S. and García, E. (2006) Making use of upper ontologies to foster interoperability between SKOS concept schemes. Online Information Review, 30(3), pp. 263-277.

Sancho, P., Martínez, I., \& Fernández-Manjón, B. (2005). Semantic Web Technologies Applied to e-learning Personalization in $<$ e-aula>. Journal of Universal Computer Science, 11(9), 1470-1481.

Santos, E. R., Boff, E., \& Vicari, R. M. (2006). Semantic web technologies applied to interoperability on an educational portal. In Proc. of Intelligent Tutoring Systems, 8th International Conference, 4053, 308-317.

Shadbolt N., Berners-Lee T., Hall W. (2006). The Semantic Web Revisited, IEEE Intelligent Systems, 21(3), 96-101, May/June. 
Sicilia M. A, García E. Sánchez-Alonso S, and Soto J. (2005). A semantic lifecycle approach to learning object repositories. In Proceedings of ELETE 2005 - eLearning on Telecommunications, Lisbon, Portugal.

Soto, J., García, E. and Sánchez-Alonso, S. (2007). Semantic learning object repositories. International Journal of Continuing Engineering Education and Life-Long Learning, 17(6), pp. 432-446.

Stokes E., Edge A., West A. (2001). Environmental education in the educational systems of the European Union, Environment Directorate-General, European Commission, April.

Tane J., Schmitz C., Stumme G., "Semantic resource management for the web: an e-learning application", in Proc. of the 13th International World Wide Web Conference, New York, NY, USA, 1-10, 2004.

Tatnall, A. (2005b), "Portals, Portals Everywhere...", in Tatnall, A. (Ed.), Web Portals: the New Gateways to Internet Information and Services, Hershey, PA, Idea Group Publishing, 1-14.

Tatnall, A., Ed. (2005a). Web Portals: the New Gateways to Internet Information and Services. Hershey, PA, Idea Group Publishing.

Tatnall, A., Ed. (2007). Encyclopaedia of Portal Technology and Applications. Hershey, PA, Information Science Reference.

Verdejo M.F., Barros B., Mayorga J.I., Read T., "Designing a Semantic Portal for Collaborative Learning Communities", in Selected Papers from the 10th Conference of the Spanish Association for Artificial Intelligence (CAEPIA03), Current Topics in Artificial Intelligence, LNCS 3040, Springer Berlin/Heidelberg, 251-259, 2004.

Vuorikari R., Manouselis N., Duval E. (2008). Using Metadata for Storing, Sharing, and Reusing Evaluations in Social Recommendation: the Case of Learning Resources, in Go D.H. \& Foo S. (Eds.) Social Information Retrieval Systems: Emerging Technologies and Applications for Searching the Web Effectively, Hershey, PA: Idea Group Publishing.

Warner, S. (1999). Internet portals, what are they and how to build a niche internet portal to enhance the delivery of information services. In Proceedings of 8th Asian-Pasific SHLL Conference.

Winkler, R. (2001). Portals - The All-In-One Web Supersites: Features, Functions, Definition, Taxonomy. SAP Design Guild, Edition 3. Retrieved May 2, 2008, http://www.sapdesignguild.org/editions/edition3/portal_definition.asp.

Woukeu A., Wills G., Conole G., Carr L., Kampa S., Hall W., "Ontological Hypermedia in Education: A framework for building web-based educational portals", in Proc. of World Conference on Educational Multimedia, Hypermedia and Telecommunications (ED-MEDIA 2003), H Honolulu, Hawaii, June 23-28, 2003. 


\section{LIST OF FIGURES}

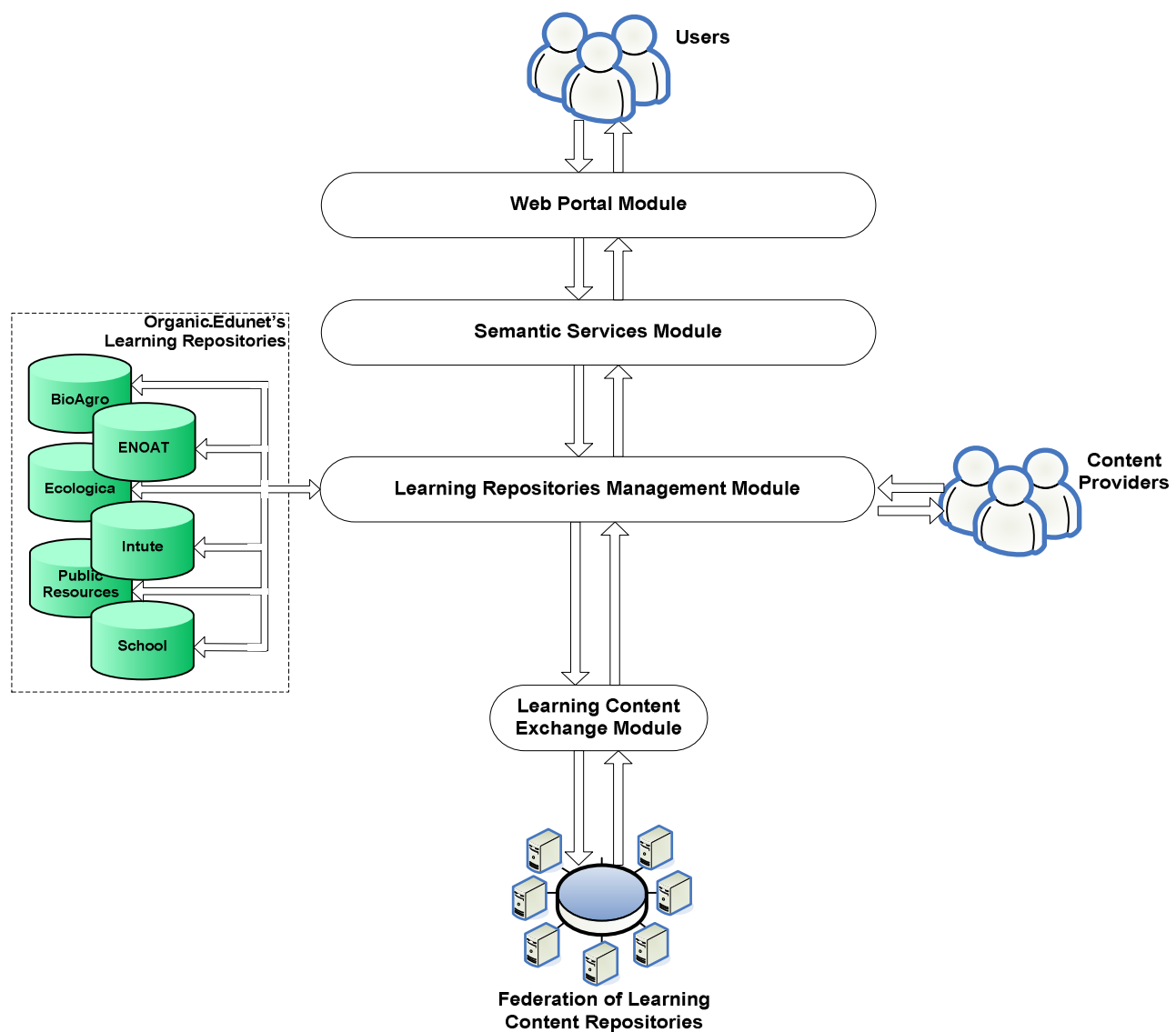

Figure 1: Overall architecture of Organic.Edunet 


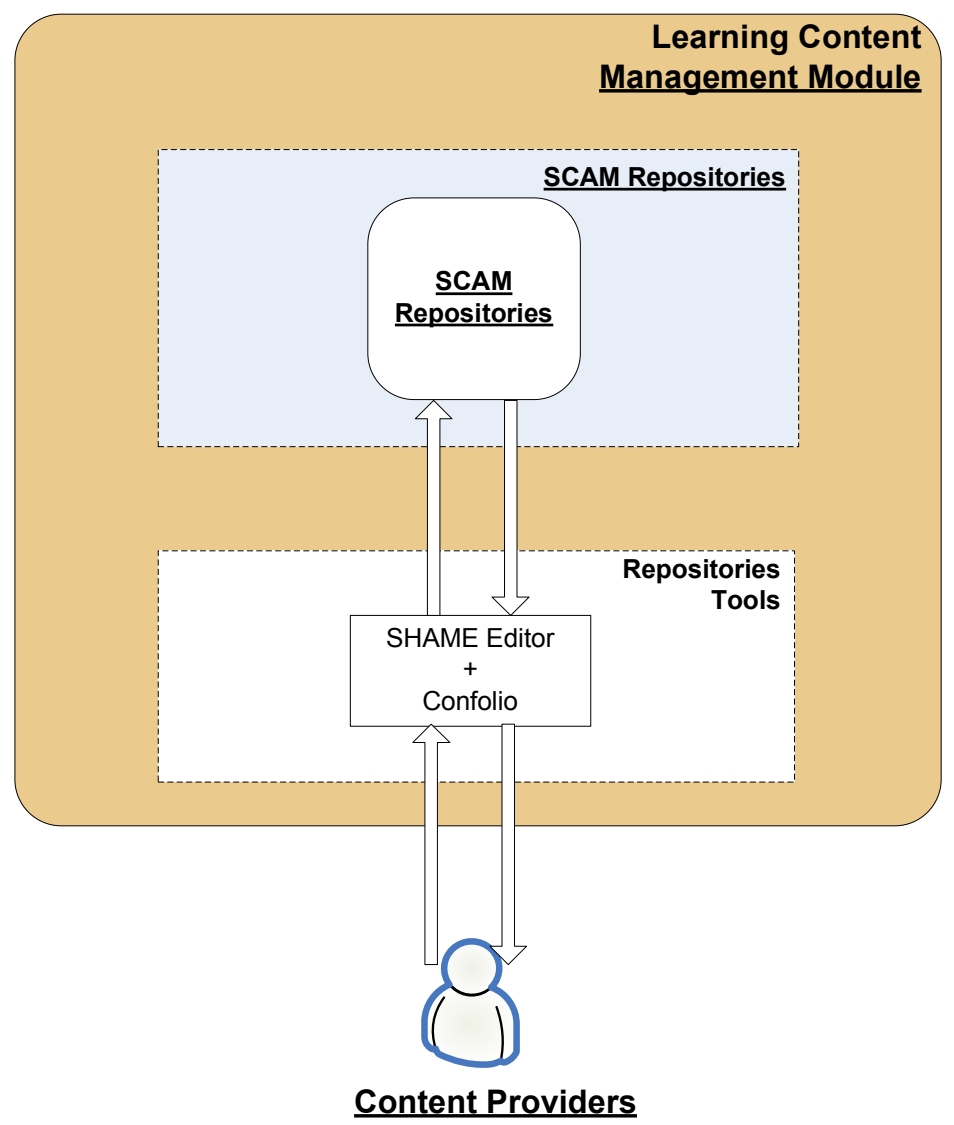

Figure 2: Overview of the Learning Repository Management Module components 


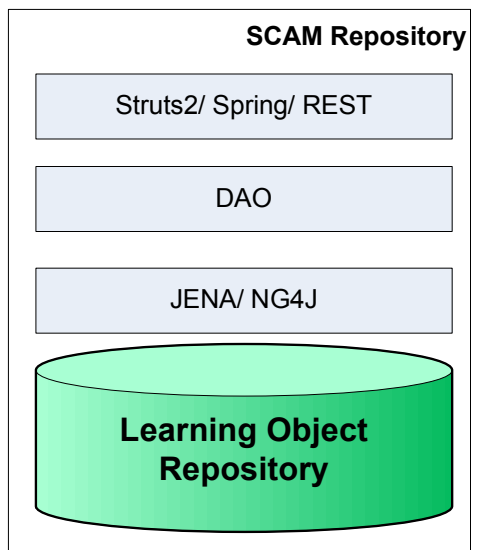

Figure 3: Overview of technology layers in a SCAM repository 


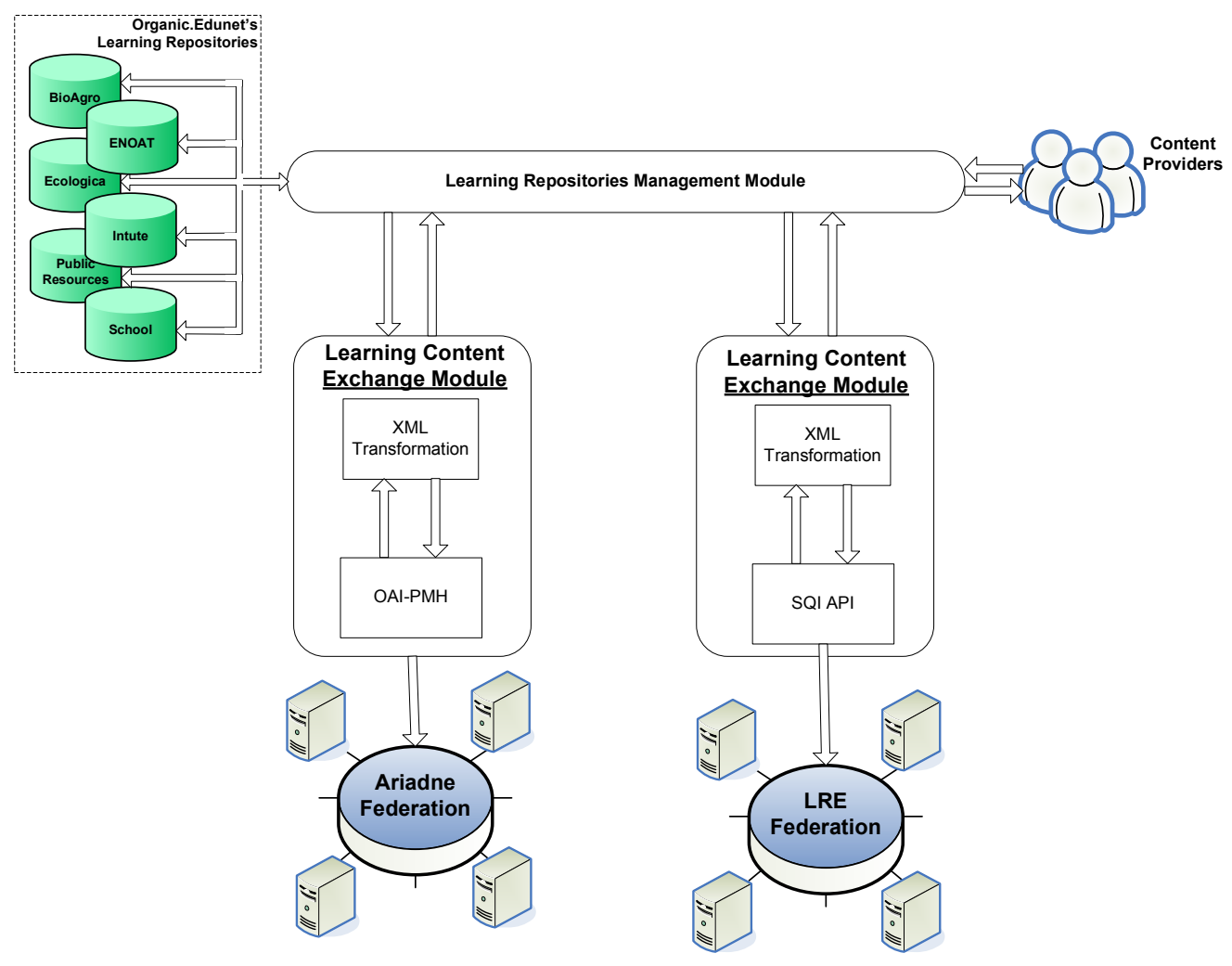

Figure 4: Illustration of the way the Learning Resources Exchange Module operates. 


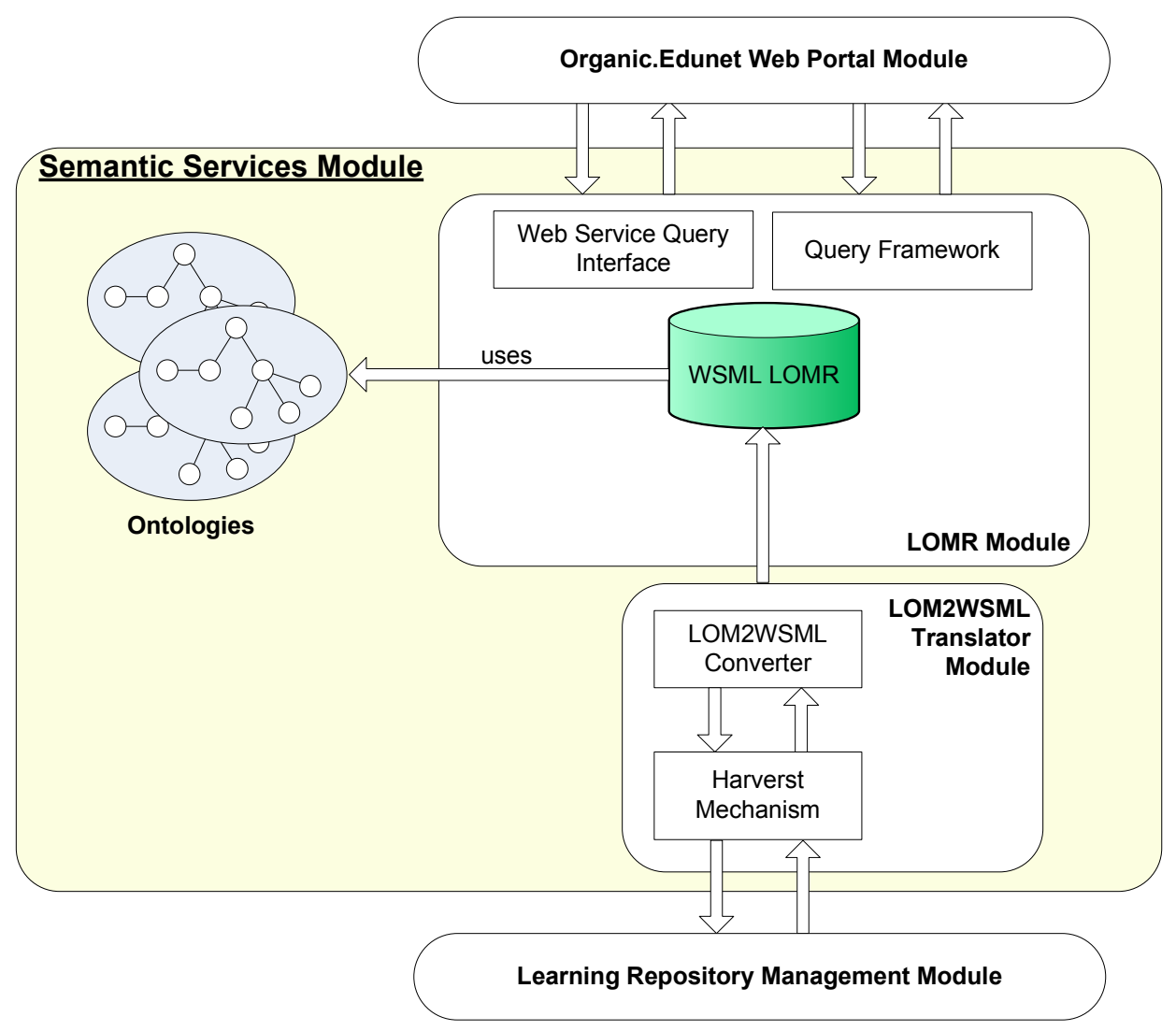

Figure 5: Overview of the Semantic Services Module 


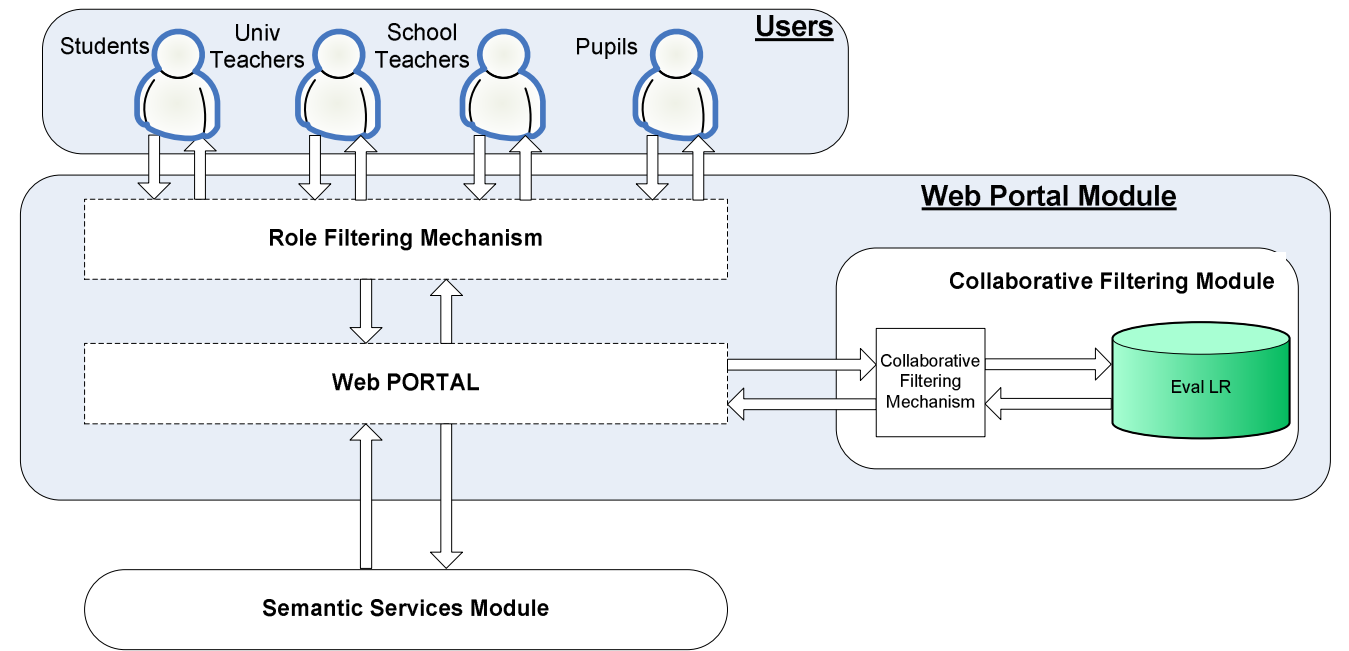

Figure 6: Elements of the Web Portal Module 


\section{Short Bios}

Dr. Nikos Manouselis is a researcher at the Greek Research \& Technology Network (GRNET S.A.). He holds a diploma in Electronics \& Computer Engineering from the Technical University of Crete, Greece (2000), a MSc in Operational Research (2002), as well as, a MSc in Electronics \& Computer Engineering (2003), both from the Technical University of Crete (TUC), Greece. In 2008, he has completed a $\mathrm{PhD}$ on applications of metadata for social information retrieval of agricultural resources, at the Informatics Laboratory of the Agricultural University of Athens (AUA).

Kostas Kastrantas holds a diploma in Electrical \& Computer Engineer from the Polytechnic School of Aristotle University of Thessaloniki, Greece (2002), and a M.Sc. in Technology Education and Digital Systems from the University of Piraeus (2007). He has strong experience in developing Web-based applications and his main interests focuses on online ontology-based and metadata.

Dr. Salvador Sanchez-Alonso obtained a university degree in Computer Science from the Pontifical University of Salamanca in 1997, and a PhD in Computing from the Polytechnic University of Madrid in 2005. He worked as an assistant professor at the Pontifical University of Salamanca for 7 years during different periods, and also as a software engineer at a software solutions company during 2000 and 2001. From 2005, he is a lecturer of the Computer Science Department of the University of Alcalá. His current research interests include learning objects reusability, metadata, Object-Oriented technologies and, Software and Web Engineering.

Jesús Cáceres obtained a university degree in Computer Science from the University of Malaga in 1992, and a MsC on education and e-learning from the University of Alcala in 2008. He has held positions as analyst applications and team leadership in several IT companies. He is currently an analyst with web applications in the Information Services at the University of Alcala and lecturer of the Computer Science department of this university. His current research interests range from learning objects technologies (LO reuse, and metadata) to software engineering and semantic web.

Hannes Ebner is a member of the Knowledge Management Research (KMR) group at the Computer Science department of the Royal Institute of Technology (KTH), Stockholm, Sweden. He holds a diploma in Telecommunication from the University of Applied Sciences, St. Pölten, Austria, as well as a MSc in Information Systems from KTH. Currently he is performing PhD studies at KTH where his focus lies on loose and non-invasive collaboration. Hannes Ebner is the main author of the collaboration server Collaborilla, and active developer within a number of Open Source projects, such as the concept browser Conzilla, the SCAM framework, and the electronic portfolio system Confolio.

Matthias Palmér has a background in mathematics and computer science. He is currently a fourth year $\mathrm{PhD}$ student in Computer Science at KTH with a focus on technology enhanced learning and Semantic Web. He is the main designer and developer of the concept browser Conzilla and the annotation tool library SHAME. He has also participated in the design and development of Collaborilla, the infrastructure Edutella, the SCAM framework and the electronic portfolio system Confolio. In his research, these projects form the basis for a prototype implementation of a learner-centric educational architecture based on open source and emerging international ICT standards.

Dr. Ambjörn Naeve (http://kmr.nada.kth.se/wiki/Amb) is the head of the Knowledge Management Research (KMR) group (http://kmr.nada.kth.se) at KTH. He is also a scientific advisor for the Centre for Sustainable Communications (SUS) at KTH (www.csc.kth.se/sustain) and the scientific director and coordinator of research on interactive learning environments at the Uppsala Learning Lab at Uppsala University (www.ull.uu.se). Since 2001 he has published more than 30 papers for international conferences and journals within the fields of knowledge management, Semantic Web, and technology enhanced learning. Within these fields he is co-editor in chief of two international journals and on the editorial board of three more, he has co-authored 5 book chapters, and co-edited 4 books and 5 special issues of international journals. Since 2003 Ambjörn Naeve has been a member of the program committee for more than 25 international workshops and conferences, including WWW, ECIS, PragWeb, IADIS, and I-Know. Within the fields of Technology Enhanced Learning, Knowledge Management and Semantic Web, the KMR group is presently participating in the European projects LUISA (www.luisa-project.eu) and Organic.Edunet (www.organic-edunet.eu). 